\title{
(6) OPEN ACCESS \\ Sidecut radius and kinetic energy: equipment designed to reduce risk of severe traumatic knee injuries in alpine giant slalom ski racing
}

\author{
Josef Kröll, ${ }^{1}$ Jörg Spörri, ${ }^{1}$ Matthias Gilgien, ${ }^{2}$ Hermann Schwameder, ${ }^{1}$ Erich Müller ${ }^{1}$
}

${ }^{1}$ Department of Sport Science and Kinesiology, University of Salzburg, Hallein-Rif, Austria ${ }^{2}$ Department of Physical Performance, Norwegian School of Sport Sciences, Oslo, Norway

\section{Correspondence to} Dr Josef Kröll, Department of Sport Science and Kinesiology, University of Salzburg, Rifer schlossallee 49, Hallein-Rif 5400, Austria;

josef.kroell@sbg.ac.at

Received 22 August 2015 Revised 4 November 2015 Accepted 12 November 2015

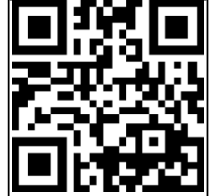

Open Access can to access more free content

\section{CrossMark}

To cite: Kröll J, Spörri J, Gilgien $\mathrm{M}$, et al. Br J Sports Med 2016;50:26-31.

\section{ABSTRACT}

Background Kinetic energy $\left(E_{\text {kin }}\right)$ increases with speed by the power of 2 and is considered a major risk factor for injuries in alpine ski racing. There is no empirical knowledge about the effect of ski geometry on $E_{k i n}$. Consequently, the aim of this study was to investigate the influence of sidecut radius on the progress of $E_{k i n}$ while skiing through a multigate section in giant slalom (GS).

Methods 5 European-Cup level athletes skied on three different pairs of GS skis varying in sidecut radii $(30,35$ and $40 \mathrm{~m}$ ). Each athlete's position over time within a six gate section (including flat and steep terrain) was captured by the use of a differential Global Navigational Satellite System. $E_{k i n}$ speed, time and path length were analysed for each pair of skis used.

Results When using skis with greater sidecut radius, average $E_{\text {kin }}$ was significantly lower over the entire six gate section, but not locally at every turn cycle.

Particular decreases of $E_{\text {kin }}$ were observed for both turns on the flat terrain, as well as for the turn at the terrain transition and the first turn on the steep terrain. The observed decreases in $E_{k i n}$ were found to be primarily explainable by increases in turn time.

Conclusions With respect to typical sport mechanisms that cause severe knee injuries, using skis with greater sidecut radius potentially provides additional injury preventative gain, particularly in specific areas within a run. However, this injury preventative gain during falls in GS should not be overestimated.

\section{INTRODUCTION}

In fall or crash situations, the magnitude of kinetic energy $\left(E_{\text {kin }}\right)$ is of particular importance since during the impact $E_{k i n}$ is dissipated over a very short distance resulting in adversely high forces. ${ }^{1}$ Since $E_{k i n}$ increases with speed by the power of 2 $\left(E_{\text {kin }}=1 / 2 \times\right.$ mass $\times$ speed $\left.^{2}\right)$, the effect of a skier's speed on injury risk seems to be considerably high. Reduced speed will obviously decrease the energy involved; therefore, 'speed in general' can be considered a major injury risk factor in alpine ski racing. ${ }^{2-4}$ However, skiing speed might not be the only factor explaining injury rates as no direct relationship was found across different competition disciplines. $^{1}$

Focusing more specifically on the knee joint as the most frequently injured body part in alpine ski racing, earlier research showed that the majority of knee injuries occur while the skier is still skiing, without any fall or crash (83\%). ${ }^{5}$ The main mechanisms for anterior cruciate ligament (ACL) injuries in alpine ski racing were found to be the 'slip-catch' and 'dynamic snowplow' activity. ${ }^{6} 7$ Based on these mechanisms, use of skis with greater sidecut radii have been suggested as a preventative measure, since these potentially help to prevent the knee positioning into excessive internal rotation and valgus. ${ }^{4-8}$

In this context, how rapidly the knee is forced into internal rotation and valgus is also important. ${ }^{7}$ Therefore, high skiing speeds and high $\mathrm{E}_{\text {kin }}$ might be driving factors as well. ${ }^{467}$ It is, furthermore, plausible that the magnitude of knee compression force, which is known to be a major component of the aforementioned mechanism, ${ }^{7}$ increases with turn speed/ $\mathrm{E}_{\mathrm{kin}}$ in the event that the ski abruptly starts carving inward. Consequently, reduced $\mathrm{E}_{\text {kin }}$ not only might help to prevent injury to athletes during fall/crash situations, but may also be an injury prevention measure with respect to the aforementioned specific injury mechanisms. ${ }^{3} 6$

To the best of our knowledge, no studies have so far addressed the influence of sidecut radius on $E_{k i n}$ in alpine ski racing. However, it is important to know how the previously suggested injury prevention measure, the sidecut radius, ${ }^{4} 8$ influences the skier's speed and therefore, $\mathrm{E}_{\text {kin }}$ in general. Owing to tactical reasons ${ }^{9}$ in skiing, it is hypothesised that the differences within each section could locally be different. ${ }^{10}$ Hence, the aim of the current study was to investigate the effect of sidecut radius on the characteristics of $\mathrm{E}_{\text {kin }}$ within a multigate section in giant slalom (GS).

\section{METHODS}

\section{Measurement protocol}

Five male European-Cup level athletes skied four runs on a 12 gate water-injected GS course with three different ski prototypes (ie, 12 runs per athlete). To ensure constant slope conditions, the experiment was conducted on three consecutive days within a particular time window of $6: 30$ to 10:30 and 24 runs/day ( 2 athletes/day). The nights prior to the test runs were characterised by temperatures between $-3^{\circ} \mathrm{C}$ and $-10^{\circ} \mathrm{C}$, and sufficient thermal radiation for snow freezing. The three different ski prototypes varied in sidecut radius $\left(30 \mathrm{~m} \bumpeq \mathrm{P}_{30}, 35 \mathrm{~m} \bumpeq \mathrm{P}_{35}, 40 \mathrm{~m} \bumpeq \mathrm{P}_{40}\right)$ based on the theoretical consideration that next to flexural and torsional stiffness, the sidecut radius affects the ski's self-steering behaviour. ${ }^{11} 12$ The most important geometrical properties of the prototypes are presented in figure 1 . All other properties were in accordance with the equipment specifications of International Ski Federation (FIS). ${ }^{13}$ To ensure the same flexural stiffness among all skis, flexural 


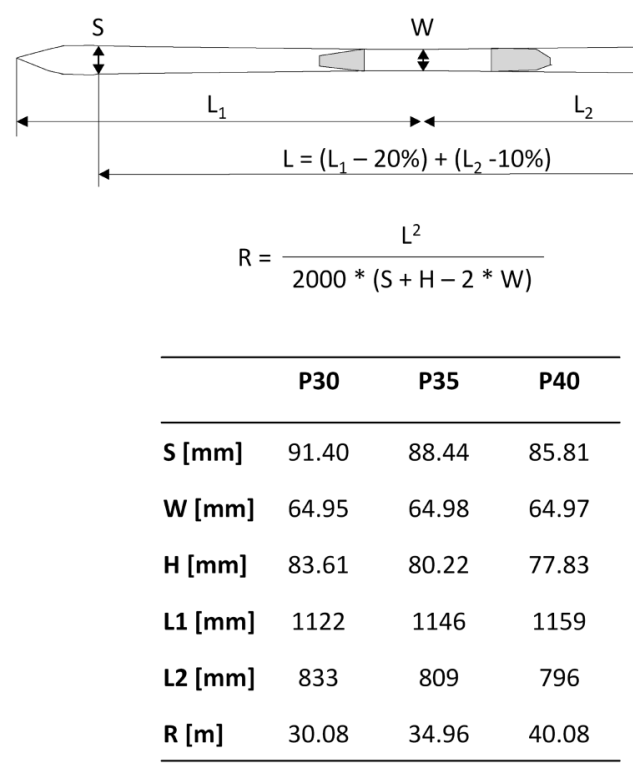

Figure 1 Main geometrical properties of $P_{30}, P_{35}$ and $P_{40}$ based on International Ski Federation specifications. ${ }^{13}$

stiffness was assessed and the results depicted no meaningful differences. In order to become familiarised to the different skis, the athletes used these skis for several months during their regular training sessions.

Per athlete and ski, a defined section of six gates within the two fastest runs was considered for further data analysis (in total 30 'six gate sections'). The six gate section had a mean gate distance of $26.7 \pm 0.8 \mathrm{~m}$ and a gate offset of $8.1 \pm 1.4 \mathrm{~m}$, and consisted of two distinct different inclinations with one gate at the convex terrain transition (figure 2).

\section{Data collection parameter computation}

The methodology used has been previously applied in several alpine skiing studies; consequently, it is only briefly described in this section. ${ }^{14-17}$ The external circumstances of the actual study were in agreement with the studies describing the accuracy of the parameters used. ${ }^{16} 17$

\section{Measurement system}

The skier's trajectory was captured by a differential global navigation satellite system (dGNSS) using GLONASS and GPS satellite signals: dual-frequency receiver $\alpha-G 3 T(50 \mathrm{~Hz}$, Javad, USA, carried in a small backpack); antenna G5Ant-2AT1 (Antcom; USA, mounted on the skier's helmet). ${ }^{18}$ The terrain geomorphology and course setting were captured using static dGNSS: dual frequency receiver $\alpha-G 3 T$ (Javad, USA); antenna GrAnt-G3T (Javad; USA). Differential position solutions of the skier trajectory, terrain and course setting were computed using additional reference data from two base stations: $\alpha$-G3T receiver (Javad, USA), GrAnt-G3T antenna (Javad, USA); and a geodetic postprocessing software (Justin, USA).

Computation of course geometry and $\mathrm{E}_{\mathrm{kin}}$

A digital terrain model was created to derive terrain inclination and course setting geometries following the same procedure as described earlier (accuracy: $0.05 \mathrm{~m}$ ). ${ }^{14} 1519$ The digital terrain model, antenna position and a pendulum model were used to approximate the centre of mass position as described and validated earlier (accuracy: position $0.09 \mathrm{~m}$, speed $0.08 \mathrm{~m} / \mathrm{s}$ ). ${ }^{17}$
The skier's instantaneous path length, speed and $\mathrm{E}_{\text {kin }}$ were derived from the centre of mass position. $\mathrm{E}_{\mathrm{kin}}$ was normalised for the skier's body weight, as performed in a previous study. ${ }^{1}$ Ground reaction force was calculated by the application of a kinetic model on the pendulum model (accuracy: 63N). ${ }^{16}$ Turns were separated by ground reaction force minima during turn switch. $^{20}$

\section{Statistical analysis}

The following steps were performed: (1) for comparison of $E_{k i n}$ at the beginning and end of the six gate section, the average values of 'Initiation Phase Turn1' and 'Completion Phase Turn6' were calculated based on a turn cycle structure definition described previously. ${ }^{9} 21$ Subsequently, average values for 'Initiation Phase Turn1' and 'Completion Phase Turn6' were tested for significant differences with two one-way repeated measures analysis of variance (ANOVAs) $\left(\mathrm{P}_{30 / 35 / 40} ; \mathrm{p}<0.05\right)$; (2) For each athlete and ski, subject mean curves of $\mathrm{E}_{\text {kin }}$ were calculated. Based on the individual subject mean curves, group mean curves were calculated and graphically visualised as mean \pm SE; (3) For the average over the entire six gate section and for the average of each turn, the significance of the differences for $E_{k i n}$, speed, time and path length was tested with a one-way repeated measure ANOVA $\left(\mathrm{P}_{30 / 35 / 40} ; \mathrm{p}<0.05\right)$, including post hoc analysis with Bonferroni correction; (4) In case of significant differences of $\mathrm{E}_{\mathrm{kin}}$ in the pairwise comparison for the entire section of the corresponding pair, the percentage differences in $\mathrm{E}_{\mathrm{kin}}\left(\%_{\mathrm{diff}} \mathrm{E}_{\mathrm{kin}}\right)$, in turn time ( $\%_{\text {diff }}$ Time) and in turn path length $(\%$ diff Length) were calculated for each turn. Subsequently, statistical testing was conducted to determine whether $\%_{\text {diff }} \mathrm{E}_{\text {kin }}$ differed significantly among the six turns by using one-way repeated measures ANOVAs $\left(\operatorname{Turn}_{1,2,3,4,5,6} ; \mathrm{p}<0.05\right)$ including a Bonferroni corrected post hoc analysis; (5) The contribution of $\%$ diff Time and $\%_{\text {diff Length to explain } \%} \%_{\text {diff }} \mathrm{E}_{\text {kin }}$ for each turn cycle was assessed by comparing the $\beta$ weights of a multiple regression analysis (enter method, $\mathrm{p}<0.05$ ).

\section{RESULTS}

\section{Beginning and end of the six gate section}

The phase average values at the beginning ('Initiation Phase Turn1') and the phase average values at the end ('Completion Phase Turn6') of the six gate section revealed no significant differences for $E_{k i n}$ when using the different skis (begin: $\mathrm{E}_{\mathrm{kinP} 30}=15.11 \pm 0.72 \mathrm{~J} / \mathrm{BW}, \mathrm{E}_{\mathrm{kinP} 35}=14.92 \pm 0.59 \mathrm{~J} / \mathrm{BW}, \mathrm{E}_{\mathrm{kinP} 40}=$ $14.71 \pm 0.58 \mathrm{~J} / \mathrm{BW}, \mathrm{p}<0.169, \quad \eta_{\mathrm{p}}^{2}=0.545 ; \quad$ end: $\mathrm{E}_{\mathrm{kinP} 30}=15.52$ $\pm 1.42 \mathrm{~J} / \mathrm{BW}, \mathrm{E}_{\mathrm{kinP} 35}=15.47 \pm 1.36 \mathrm{~J} / \mathrm{BW}, \quad \mathrm{E}_{\mathrm{kinP} 40}=15.22 \pm 1.72$ $\left.\mathrm{J} / \mathrm{BW}, \mathrm{p}<0.752, \eta_{\mathrm{p}}^{2}=0.173\right)$.

\section{Entire six gate section}

The time course of $\mathrm{E}_{\text {kin }}$ for the three analysed skis are presented in figure 3 . As a general trend, $E_{\text {kin }}$ decreases during Turn 2 and Turn3 (ie, prior and during the terrain transition), and tends to increase along the subsequent turns. Within the turns, $\mathrm{E}_{\text {kin }}$ starts with a local maximum at turn switch and decreases during the turn cycle to a local minimum around gate passage, before increasing again until the subsequent turn switch. Comparing the skis, one can observe pronounced differences for both turns in the flat terrain, as well as for the turn at the terrain transition with increasing sidecut radius.

As shown in table 1, this results in significant differences in the entire six gate section averages of $\mathrm{E}_{\text {kin }}$, speed and time between $\mathrm{P}_{30}$ and $\mathrm{P}_{35}$, and between $\mathrm{P}_{30}$ and $\mathrm{P}_{40}$. No significant differences were observed for path length. Post hoc comparison 
Figure 2 On-hill measurement setup. Left: Schematic illustration of the six gate section. Right bottom: Illustration of the course setting characteristics, characterised by gate distance (GD) and gate offset (GO). ${ }^{14}$ Right top: Values of the course setting characteristics and the slope inclinations within the analysed six gate section.

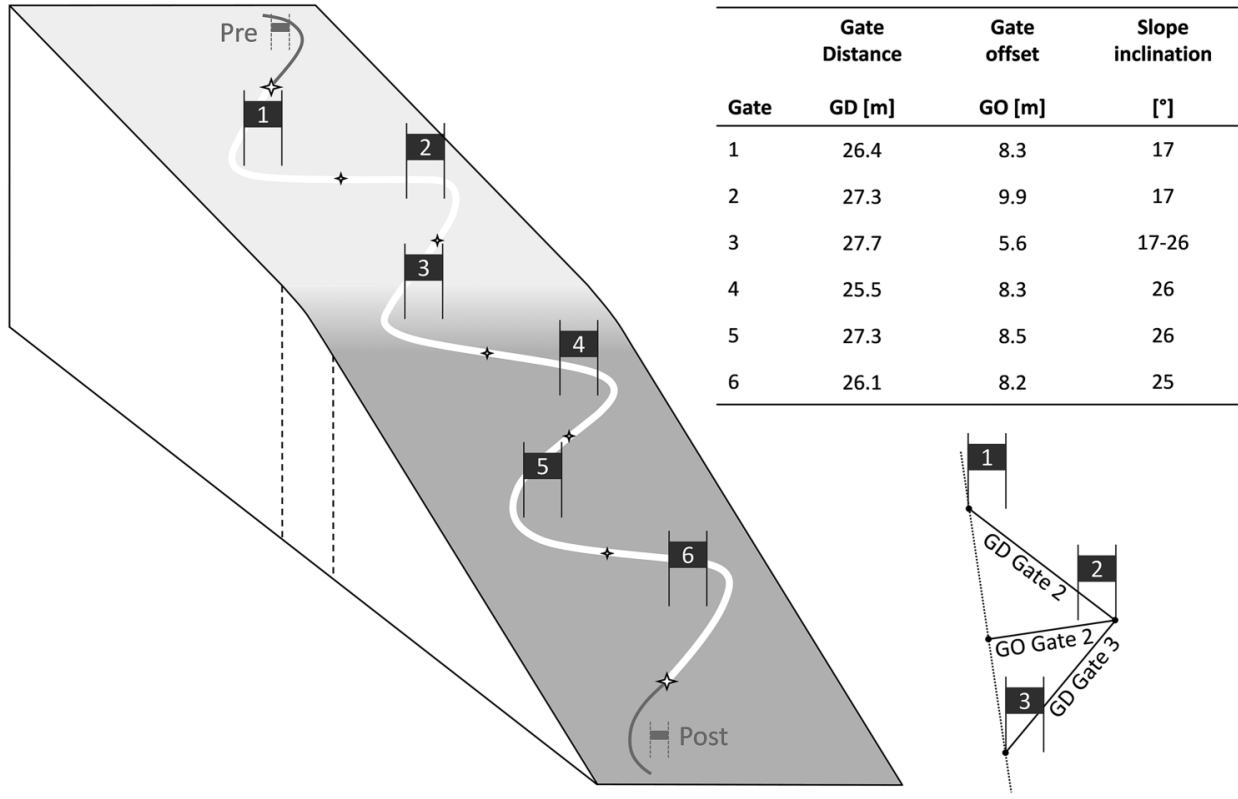

of the entire six gate section did not reveal significant differences between $\mathrm{P}_{35}$ and $\mathrm{P}_{40}$.

\section{Turn by turn analysis}

The results for $\mathrm{E}_{\text {kin }}$ separated into the single turns are presented in table 2. Significant differences between the tested skis were revealed for the turns in the flat terrain (Turn1 and Turn2), the turn at the terrain transmission and the first turn in the steep terrain, but not for Turn5 and Turn6. In the pairwise comparisons, different turns showed strong effects. When comparing $\mathrm{P}_{30}$ with $\mathrm{P}_{35}$, Turn2, Turn3 and Turn4 were mainly affected; while comparing $\mathrm{P}_{30}$ with $\mathrm{P}_{40}$, Turn1, Turn2 and Turn 3 showed significant differences. The most pronounced differences were observed for $\mathrm{P}_{30}$ vs $\mathrm{P}_{40}\left(8.77 \%\right.$ decrease in $\mathrm{E}_{\text {kin }}$ for Turn2).

The $\%_{\text {diff }} \mathrm{E}_{\text {kin }}$ between $\mathrm{P}_{30}$ and $\mathrm{P}_{35}$, as well as between $\mathrm{P}_{30}$ and $\mathrm{P}_{40}$, are illustrated in figure 4 . Within the six gate section, the $\%_{\text {diff }} E_{\text {kin }}$ revealed a global tendency for $\mathrm{P}_{30}$ vs $\mathrm{P}_{40}$ $\left(\mathrm{p}<0.137, \eta_{\mathrm{p}}^{2}=0.375\right)$, but not for $\mathrm{P}_{30}$ vs $\mathrm{P}_{35} \quad(\mathrm{p}<0.053$, $\left.\eta_{\mathrm{p}}^{2}=0.505\right)$. A selective post hoc analysis for $\mathrm{P}_{30}$ vs $\mathrm{P}_{40}$ comparing the lowest value within the six gate section (ie, Turn2) with all other turns revealed a tendency for Turn $4(\mathrm{p}<0.077)$ and

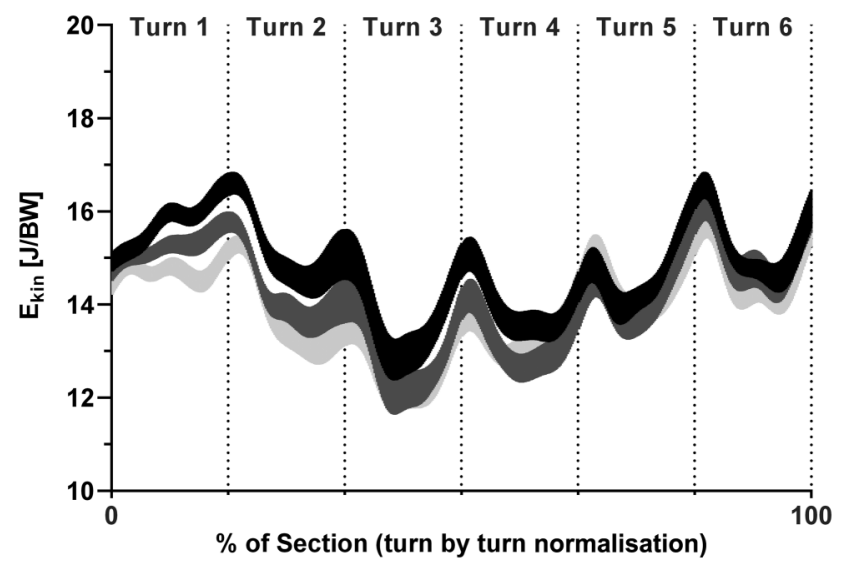

Figure 3 Areas of uncertainty around the estimate of the mean $( \pm \mathrm{SE})$ illustrating $E_{\text {kin }}$ for the entire six gate section. Vertical dotted lines separate the turns. (black $\triangleq \mathrm{P}_{30}$; dark grey $\triangleq \mathrm{P}_{35}$; light grey $\triangleq \mathrm{P}_{40}$ ). significant differences for Turn5 $(\mathrm{p}<0.012)$ and Turn6 $(\mathrm{p}<0.030)$.

The results of the multiple regression analysis assessing the contribution of $\%_{\text {diff }}$ Time and $\%_{\text {diff }}$ Length to explain the $\%_{\text {diff- }}$ $\mathrm{E}_{\mathrm{kin}}$ are presented in figure 5. For all situations significant prediction models emerged, explaining between $96.5 \%$ and $99.8 \%$ of the variance. Comparing the $\beta$ weights, it becomes obvious that for all conditions, $\%_{\text {diff }}$ Time was distinctly more relevant for predicting $\%_{\text {diff }} \mathrm{E}_{\text {kin }}$ than $\%_{\text {diff }}$ Length.

\section{DISCUSSION}

The main findings of this study were: (1) compared to $\mathrm{P}_{30}$, average $\mathrm{E}_{\mathrm{kin}}$ within a six gate section decreased for skis with greater sidecut radius $\left(\mathrm{P}_{30} \rightarrow \mathrm{P}_{35}=-4.1 \% ; \mathrm{P}_{30} \rightarrow \mathrm{P}_{40}=-5.6 \%\right) ;(2)$ decreases in $\mathrm{E}_{\text {kin }}$ were found to be specifically located and not constant within the entire six gate section; (3) the observed decrease in $\mathrm{E}_{\mathrm{kin}}$ can primarily be explained by differences in turn time rather than by differences in path length, which remained constant.

\section{Methodological considerations}

Although the use of a non-invasive technology (dGNSS) serves a number of advantages (eg, use of athletes' personal equipment, no additional disturbing instrumentation), the method used in this study has one major limitation: it corresponds to a whole body approach and therefore, it is not suitable to determine knee load components (eg, compression, ab/adduction, rotation loads). Considering this limitation, $\mathrm{E}_{\mathrm{kin}}$ on a full body level possibly does not necessarily correlate with the local joint risk factors. Moreover, due to the whole body approach it is not possible to provide more in-depth explanations on a skier's movements (eg, edging angles).

For future research, additional efforts should be made to combine the method used (dGNNS) with multisegment kinematics, as has been previously suggested. ${ }^{22}$ Such research could provide more information needed to improve design and aid in injury prevention. ${ }^{23}$ Despite the limitation of the used method and the discussion, therefore, being on a rather superficial level, the study investigates for the first time the effect of equipment design on $E_{\text {kin }}$ within a multigate section. 
Table 1 Descriptive and inferential statistics for group mean $\pm S D$ of the entire six gate section averages: the kinetic energy $\left(E_{k i n}\right)$, turn speed (Speed), time for the section (Time) and skiing distance (Path length) of skiers are compared for the tested skis $\left(P_{30}, P_{35}, P_{40}\right)$

\begin{tabular}{|c|c|c|c|c|c|c|c|c|}
\hline & \multicolumn{3}{|c|}{ Group mean \pm SD } & \multicolumn{2}{|l|}{ ANOVAt } & \multicolumn{3}{|c|}{ Pairwise comparisons } \\
\hline & $\mathbf{P}_{30}$ & $\mathbf{P}_{35}$ & $\mathbf{P}_{40}$ & p Value & $\eta_{\mathrm{p}}^{2}$ & $\mathbf{P}_{30} / \mathbf{P}_{35}(\%)$ & $P_{30} / P_{40}(\%)$ & $P_{35} / P_{40}$ \\
\hline$E_{\text {kin }}(J / B W)$ & $14.77 \pm 0.82$ & $14.16 \pm 0.80$ & $13.95 \pm 0.78$ & $0.000 * * *$ & 0.904 & $-4.10^{*}$ & $-5.56^{* *}$ & \\
\hline Speed $(\mathrm{m} / \mathrm{s})$ & $17.00 \pm 0.48$ & $16.65 \pm 0.47$ & $16.52 \pm 0.46$ & $0.000 * * *$ & 0.902 & $-2.09 *$ & $-2.83^{* *}$ & \\
\hline Time (s) & $9.68 \pm 0.20$ & $9.90 \pm 0.19$ & $9.98 \pm 0.20$ & $0.000^{* * *}$ & 0.920 & $2.23^{* *}$ & $3.01^{* *}$ & \\
\hline Path length (m) & $201.74 \pm 3.33$ & $201.85 \pm 3.45$ & $201.96 \pm 3.51$ & $0.296^{\mathrm{ns}}$ & 0.266 & & & \\
\hline
\end{tabular}

Additionally, it provides fundamental knowledge about the tactical behaviour of skiers and suggests effective prevention measures; even so, these findings need further verification by epidemiological studies.

\section{The effect of sidecut radius on skiing tactic Velocity barrier}

Skiing tactic is one possibility to modulate instantaneous $\mathrm{E}_{\text {kin }}$ and can be understood as the choice of a specific technique (carving or skidding) in combination with the choice of line. ${ }^{10} 21 \quad 2425$ Attributable to tactical reasons, in some cases even a lower instantaneous/section performance can be beneficial if it results in a disproportionally higher performance in the following section. ${ }^{9} 2126$

From a skiing tactic point-of-view, the so called 'velocity barrier' seems to play a major role $;^{26-28}$ from a performance perspective, a high amount of potential energy should be transformed as fast as possible into $\mathrm{E}_{\mathrm{kin}}{ }^{21}{ }^{27}$ However, even fast skiers will need to dissipate excess $\mathrm{E}_{\text {kin }}$ at certain points along the course $\mathrm{e}^{28}$ as otherwise they would become more prone to making mistakes. This intuitive control of speed by the skiers in order to avoid mistakes is called 'velocity barrier'. ${ }^{26}$ The actual study shows that during the six gate section, the complete potential energy was dissipated because the $\mathrm{E}_{\text {kin }}$ at the beginning and at the end of this section was essentially the same. This is most likely explainable by the 'velocity barrier'.

The phenomenon of the 'velocity barrier' also might help to explain the differences in $\mathrm{E}_{\mathrm{kin}}$ between the different skis varying in sidecut radius. Skis with greater sidecut radii are theoretically associated with a decreased self-steering effect, which plausibly influences the performance. ${ }^{11} 12$
Comparing $\mathrm{P}_{30}-\mathrm{P}_{40}$ (figure 4 , right), the skiers were only able to utilise the beneficial effect of the smaller sidecut radius for the flat terrain of the analysed section and for the turn at the terrain transition, resulting in $\mathrm{E}_{\mathrm{kin}}$ differences at these gates. Within the last three gates, the inclination and therefore the amount of convertible potential energy might have been too high. If all of the available potential energy had been transformed into $E_{k i n}$, the skiers would have been more prone to making mistakes. Instead of using the smaller sidecut radius to perform purely carved turns with a minimum of energy dissipation, they might have controlled their speed by intuitively increasing their amount of skidding. Thus, the 'velocity barrier' might also serve as an explanation for the differences in $\mathrm{E}_{\mathrm{kin}}$ between skis with different sidecut radii.

How turn time and path length influences $E_{k i n}$

As found in this study, the differences in $E_{k i n}$ can be explained primarily by differences in turn time. This is in accordance with a previous study in GS showing pronounced differences in the skier's speed and turn time, but only slight differences in path length. ${ }^{25}$ Furthermore, alterations in $\mathrm{E}_{\text {kin }}$ are known to be mainly dependent on ski-snow friction, since energy dissipation due to air drag only marginally contributes to the total loss of energy in GS. ${ }^{25}$

If the influence of air drag on energy dissipation is negligible and path length is the same, as observed in this study, by definition, ski-snow friction must be the major factor. In this context, the current study revealed that in sections with flat terrain or during terrain transitions, $\mathrm{E}_{\mathrm{kin}}$ was higher while skiing on $\mathrm{P}_{30}$ than while skiing on either $\mathrm{P}_{35}$ or $\mathrm{P}_{40}$. Based on these findings, one could presume that in the aforementioned sections, skiers

Table 2 Descriptive and inferential statistics for skier kinetic energy $\left(E_{k i n}\right)$ group mean $\pm S D$ of each turn average for the tested skis $\left(P_{30}, P_{35}\right.$ $\left.\mathrm{P}_{40}\right)$

\begin{tabular}{|c|c|c|c|c|c|c|c|}
\hline \multirow[b]{2}{*}{$E_{\text {kin }}(J / B W)$} & \multicolumn{3}{|c|}{ Group mean \pm SD } & \multicolumn{2}{|l|}{ ANOVAt } & \multicolumn{2}{|c|}{ Pairwise comparisons } \\
\hline & $P_{30}$ & $P_{35}$ & $\mathbf{P}_{40}$ & $\mathrm{p}$ Value & $\eta_{\mathrm{p}}^{2}$ & $\mathrm{P}_{30} / \mathrm{P}_{35}(\%)$ & $\mathrm{P}_{30} / \mathrm{P}_{40}(\%)$ \\
\hline Turn 1 & $15.76 \pm 0.72$ & $15.23 \pm 0.67$ & $14.72 \pm 0.53$ & $0.004^{* *}$ & 0.838 & & $-6.61^{*}$ \\
\hline Turn 2 & $15.19 \pm 0.98$ & $14.28 \pm 0.89$ & $13.86 \pm 0.92$ & $0.000^{* * *}$ & 0.904 & $-6.03^{* *}$ & $-8.77^{* *}$ \\
\hline Turn 3 & $13.73 \pm 1.37$ & $12.87 \pm 1.14$ & $12.60 \pm 0.82$ & $0.012^{* *}$ & 0.687 & $-6.62^{(t)}$ & $-8.25^{(t)}$ \\
\hline Turn 4 & $13.97 \pm 1.06$ & $13.21 \pm 1.04$ & $13.32 \pm 0.86$ & $0.004^{* *}$ & 0.641 & $-5.45^{*}$ & \\
\hline Turn 5 & $14.67 \pm 0.96$ & $14.28 \pm 1.03$ & $14.38 \pm 1.09$ & $0.212^{\text {ns }}$ & 0.334 & & \\
\hline Turn 6 & $15.29 \pm 0.90$ & $15.11 \pm 1.21$ & $14.81 \pm 1.23$ & $0.246^{\mathrm{ns}}$ & 0.303 & & \\
\hline
\end{tabular}



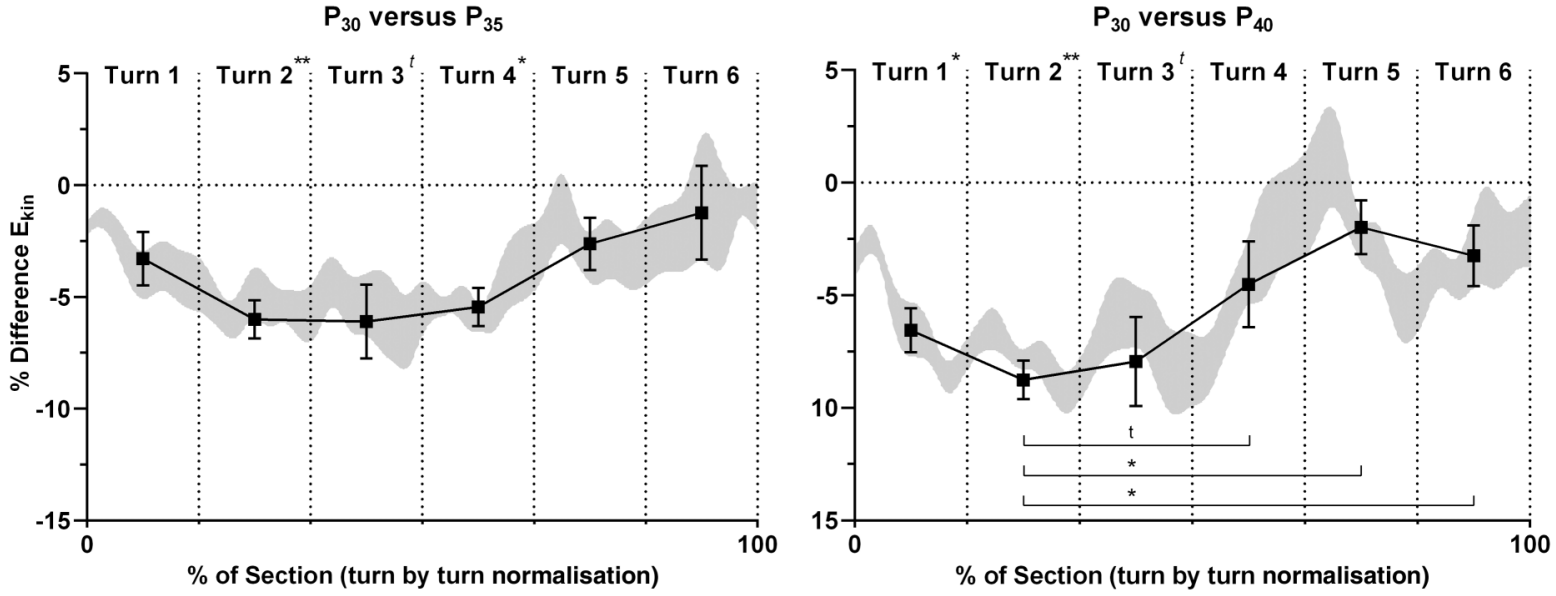

Figure 4 Percentage differences in kinetic energy $\left(\%_{\text {diff }} E_{k i n}\right)$ between $P_{30}$ and $P_{35}$ (Left), and between $P_{30}$ and $P_{40}$ (Right). Black squares show Mean $\pm S E$ for each single turn. The grey curves show the instantaneous areas of uncertainty around the estimate of the mean ( \pm SE). The bars across

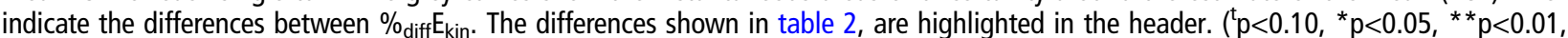
$* * * p<0.001)$.

gained more speed while skiing on $\mathrm{P}_{30}$. This can be explained by the increased self-steering effect of skis with smaller sidecut radius, since this effect is expected to result in less ski-snow friction due to less skidding. ${ }^{11}{ }^{29}$ However, in the subsequent steep terrain, it is reasonable that skiers generate higher ski-snow friction with $\mathrm{P}_{30}$ than with $\mathrm{P}_{35}$ and $\mathrm{P}_{40}$, since the relative differences in $\mathrm{E}_{\mathrm{kin}}$ were found to decrease again (figure 4); One explanation for these observations might be based on the 'velocity barrier' described above.

\section{The effect of sidecut radius on injury prevention aspects related to $\mathrm{E}_{\mathrm{kin}}$}

The role of $E_{k i n}$ in fall/crash situations

Recent data show that 59\% of all injuries result from fall or crash situations, either from the initial impact or the subsequent tumbling/sliding. ${ }^{5} \mathrm{E}_{\text {kin }}$ might be particularly important for non-lower-extremity injuries, since more than 90\% of these occur while falling. ${ }^{5}$ Experiments with safety barriers depicted that acceleration values on an instrumented dummy were linearly correlated with the impact speed. ${ }^{30}$ A study comparing different disciplines showed that $\mathrm{E}_{\mathrm{kin}}$ is almost doubled from GS to downhill. ${ }^{1}$ Thus, the observed reduction in $\mathrm{E}_{\mathrm{kin}}$ for greater sidecut radius in GS (on average 4.1\% for $\mathrm{P}_{30}$ and 5.6\% for $\mathrm{P}_{40}$; up to $9 \%$ in specific phases) provides only a marginal injury prevention advantage during fall or crash situations compared to $\mathrm{E}_{\mathrm{kin}}$ reduction efforts while skiing downhill.

The role of $E_{\text {kin }}$ in typical mechanisms of severe knee injuries The major injury preventative effects of skis with greater sidecut radius (ie, the reduction of the ski's aggressiveness and selfsteering effect) have been presented by other studies within this special issue and these, therefore, will not be discussed here. $^{31} 32$

Typical mechanisms for ACL injuries in alpine ski racing are characterised as follows: during an out-of-balance situation the inside edge of one ski abruptly catches the snow surface thus forcing the knee into internal rotation and valgus, and resulting in high knee compression forces because the direction of
$P_{30}$ versus $P_{35}$

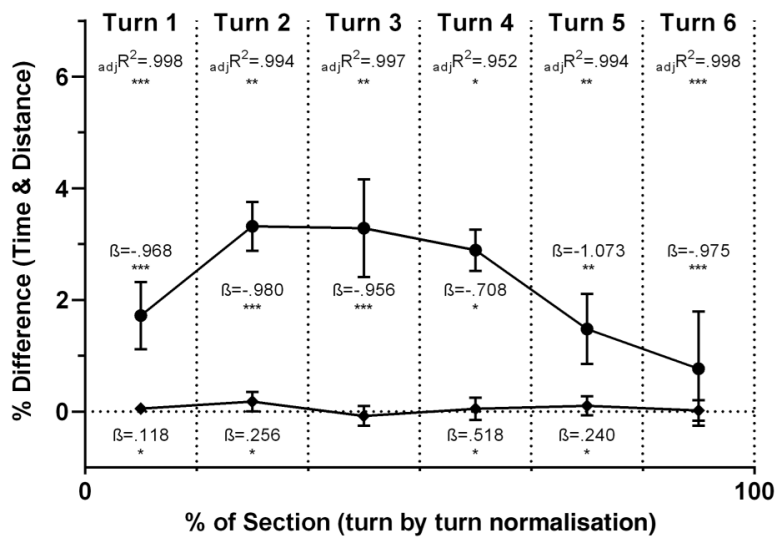

$P_{30}$ versus $P_{40}$

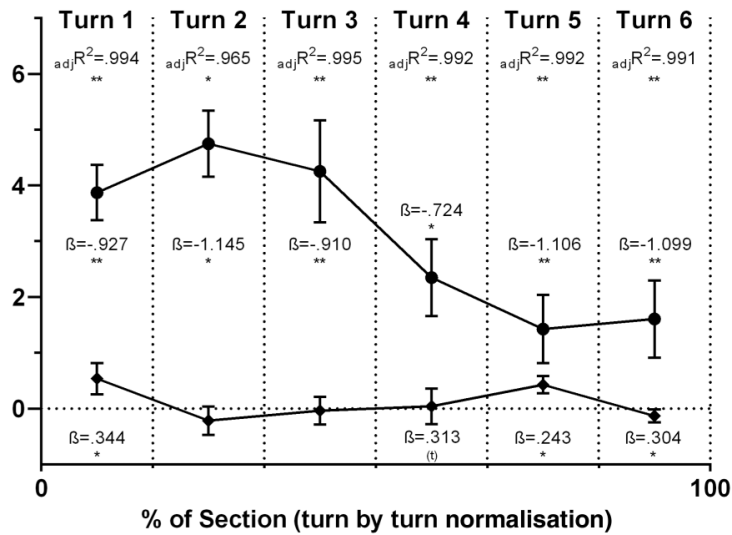

Figure 5 Mean \pm SE percentage difference for turn time (circles) and path length (diamonds). Left: between $\mathrm{P}_{30}$ and $\mathrm{P}_{35 ;}$ Right: between $\mathrm{P}_{30}$ and $P_{40}$. In case of significance of the multiple regression model, for all turns the explanation rate $\left({ }_{\text {adj }} R^{2}\right)$ and $\beta$-weights of turn time and path length are reported. $\left({ }^{t} p<0.1,{ }^{*} p<0.05,{ }^{* *} p<0.01,{ }^{* *} p<0.001\right)$. 
centre of mass and the direction of ski diverge. ${ }^{6} 7$ In this context, it is known that the lateral displacement of the ski and therefore the magnitude of internal rotation takes place until the skier can initiate a compensatory movement depends on the edge angle, the ski's sidecut radius, the ski's bending characteristics and the skier's speed $\left(E_{k i n}\right){ }^{33}$ When catching the edge while recovering from an out-of-balance situation, the edge angle cannot actively be altered by the skier. Moreover, the ski's sidecut radius and bending characteristics are given constraints that cannot be changed while skiing. Thus, the only remaining factor to decrease the lateral displacement of the ski and the magnitude of internal rotation during the injury situation is to decrease the skier's speed $\left(\mathrm{E}_{\mathrm{kin}}\right)$. Consequently, the sidecut-induced reduction in speed $\left(\mathrm{E}_{\text {kin }}\right)$ observed in the current study might be considered an additional injury preventative gain. Furthermore, the decrease in speed $\left(\mathrm{E}_{\mathrm{kin}}\right)$ might also reduce the magnitude of knee compression force during the injury mechanism, since the skier's loading during turns is known to be mainly dependent on turn speed and turn radius. $^{1}$

Finally, it has to be pointed out that the reported differences were mainly observed for the two turns in the flat terrain where speed $\left(\mathrm{E}_{\mathrm{kin}}\right)$ was relatively high. Consequently, it can reasonably be assumed that the location of the depicted differences is meaningful with regard to severe knee injuries prevention in GS.

\section{CONCLUSION}

To discuss the injury preventative gains from the observed reduction in $E_{k i n}$ as related to severe traumatic knee injuries, one should distinguish between fall/crash situations and the typical injury mechanisms while skiing: with respect to fall or crash situations, it can be concluded that in GS the injury preventative gain from increased sidecut radius should not be overestimated, since GS innately has distinct lower $\mathrm{E}_{\text {kin }}$ compared to other disciplines. With respect to the contribution of greater sidecut radius on the typical mechanisms of ACL injuries, potential preventative gains might only be related to specific locations of the six gate section (ie, the turns in the flat terrain and at the terrain transition). At these locations, high speed $\left(\mathrm{E}_{\mathrm{kin}}\right)$ plausibly contributes to unexpected lateral displacement when a ski starts carving inward after catching the edge in an out-of-balance situation.

\section{What are the findings?}

- When skiing on skis with increased sidecut radius within a six gate section, on average, (kinetic energy) $E_{k i n}$ is decreased by up to $5.6 \%$. However, due to skiing tactic reasons (ie, 'velocity barrier') this is mainly because of differences at specific turns (local differences: up to 8.8\%).

- Regarding fall or crash situations in giant slalom, the injury preventative gain from skis with greater sidecut radius with regard to severe traumatic knee injuries should not be overestimated.

- With respect to the typical injury mechanisms resulting in severe knee injuries in giant slalom, the observed sidecut induced reduction in speed and $E_{k i n}$ may provide additional injury preventative gains, at least within specific sections off the course.

\section{How might it impact on clinical practice in the future?}

- This study investigates for the first time the influence of an equipment-based prevention measure (the skis sidecut radius) for risk of injury from the external factor kinetic energy.

- It provides deeper knowledge on how kinetic energy develops within a multigate section.

Contributors JS, JK and EM conceptualised and coordinated the study. JK, JS and MG contributed to study design and data collection. MG conducted data processing. $\mathrm{JK}, \mathrm{JS}$ and $\mathrm{HS}$ analysed the data. All authors contributed to the intellectual content, writing of the manuscript and approved its content.

Funding This study was financially supported by the International Ski Federation (FIS) and the St Moritz Health and Innovation Foundation. The funding sources had no involvement in the collection, analysis and interpretation of the data, in the writing of the report, or in the decision to submit this paper for publication.

Competing interests None declared.

Ethics approval This study was approved by the Ethics Committee of the Department of Sport Science and Kinesiology at the University of Salzburg.

Provenance and peer review Not commissioned; externally peer reviewed.

Open Access This is an Open Access article distributed in accordance with the Creative Commons Attribution Non Commercial (CC BY-NC 4.0) license, which permits others to distribute, remix, adapt, build upon this work non-commercially, and license their derivative works on different terms, provided the original work is properly cited and the use is non-commercial. See: http://creativecommons.org/ licenses/by-nc/4.0/

\section{REFERENCES}

1 Gilgien M, Spörri J, Kröll J, et al. Mechanics of turning and jumping and skier speed are associated with injury risk in men's World Cup alpine skiing: a comparison between the competition disciplines. Br J Sports Med 2014:48:742-7.

2 Flørenes TW, Bere T, Nordsletten L, et al. Injuries among male and female World Cup alpine skiers. Br J Sports Med 2009;43:973-8.

3 Bere T, Flørenes TW, Krosshaug T, et al. Events leading to anterior cruciate ligament injury in World Cup Alpine Skiing: a systematic video analysis of 20 cases. Br J Sports Med 2011;45:1294-302.

4 Spörri J, Kröll J, Amesberger G, et al. Perceived key injury risk factors in world cup alpine ski racing-an explorative qualitative study with expert stakeholders. $\mathrm{Br} J$ Sports Med 2012;46:1059-64.

5 Bere T, Flørenes TW, Krosshaug T, et al. A systematic video analysis of 69 injury cases in World Cup alpine skiing. Scand J Med Sci Sports 2014;24:667-77.

6 Bere T, Flørenes TW, Krosshaug T, et al. Mechanisms of anterior cruciate ligament injury in World Cup alpine skiing: a systematic video analysis of 20 cases. Am J Sports Med 2011:39:1421-9.

7 Bere $\mathrm{T}$, Mok KM, Koga $\mathrm{H}$, et al. Kinematics of anterior cruciate ligament ruptures in World Cup alpine skiing: 2 case reports of the slip-catch mechanism. Am J Sports Med 2013;41:1067-73.

8 Kröll J, Spörri J, Gilgien M, et al. The process of injury prevention within an international sport federation-Elite Alpine ski racing as an example. Sport Orthop Traumatol 2013;29:288-96.

9 Spörri J, Kröll J, Schwameder $\mathrm{H}$, et al. Course setting and selected biomechanical variables related to injury risk in alpine ski racing: an explorative case study. $\mathrm{Br} J$ Sports Med 2012;46:1072-7.

10 Supej M, Cernigoj M. Relations between different technical and tactical approaches and overall time at men's World Cup giant slalom races. Kinesiologia Slovenica 2006;12:63-9.

11 LeMaster R. Ultimate skiing. Leeds, UK: Human Kinetics, 2010. 212 p.

12 Howe J. The new skiing mechanics. 2nd edn. Waterford: McIntire Publishing, 2001.

13 International Ski Federation FIS. Specification for competition equipment and comercial markings. Edition 2011/12 ed. Oberhofen, Switzerland: Self-publisher, 2011.

14 Gilgien M, Crivelli P, Spörri J, et al. Characterization of course and terrain and their effect on skier speed in World Cup alpine ski racing. PLOS ONE 2015;10:e0118119.

15 Gilgien M, Crivelli P, Spörri J, et al. Correction: characterization of course and terrain and their effect on skier speed in world cup alpine ski racing. PLOS ONE 2015;10:e0128899.

16 Gilgien M, Spörri J, Chardonnens J, et al. Determination of external forces in alpine skiing using a differential global navigation satellite system. Sensors 2013;13:9821-35. 
17 Gilgien M, Spörri J, Chardonnens J, et al. Determination of the centre of mass kinematics in alpine skiing using differential global navigation satellite systems. J Sports Sci 2015;33:960-9.

18 Gilgien M, Spörri J, Limpach P, et al. The effect of different Global Navigation Satellite System methods on positioning accuracy in elite alpine skiing. Sensors 2014;14:18433-53.

19 Gilgien M. Characterisation of Skiers' Mechanics, Course Setting and Terrain Geomorphology in World Cup Alpine Skiing using Global Navigation Satellite Systems: Injury Risk, Performance and Methodological Aspects [PhD Thesis]. Norwegian School of Sport Sciences Oslo; 2014. ISBN: 978-82-502-0500-0.

20 Spörri J, Kröll J, Haid C, et al. Potential mechanisms leading to overuse injuries of the back in alpine ski racing: a descriptive biomechanical study. Am J Sports Med 2015:43:2042-8.

21 Spörri J, Kröll J, Schwameder $\mathrm{H}$, et al. Turn characteristics of a top world class athlete in giant slalom: a case study assessing current performance prediction concepts. Int J Sport Sci Coach 2012;7:647-59.

22 Supej M. 3D measurements of alpine skiing with an inertial sensor motion capture suit and GNSS RTK system. J Sports Sci 2010;28:759-69.

23 Schott W, Senner V, Johnson RJ, et al. Emergency release for winter sports equipment. J ASTM Int 2010;7:102824.

24 Lind D, Sanders SP. The physics of skiing - skiing at the triple point. New York: Springer Verlag, 2004

25 Supej M, Saetran L, Oggiano L, et al. Aerodynamic drag is not the major determinant of performance during giant slalom skiing at the elite level. Scand J Med Sci Sports 2013;23:e38-47.
26 Supej M, Kipp R, Holmberg HC. Mechanical parameters as predictors of performance in alpine World Cup slalom racing. Scand J Med Sci Sports 2011;21: e72-81.

27 Supej M. Differential specific mechanical energy as a quality parameter in racing alpine skiing. J Appl Biomech 2008;24:121-9.

28 Reid R, Gilgien M, Moger $T$, et al. Turn characteristics and energy dissipation in slalom. In: Müller E, Lindinger S, Stöggl T, eds. Science and Skiing IV-Proceedings of the 4th International Congress on Science and Skiing (ICSS). Maidenhead, UK: Meyer \& Meyer Sport (UK) Ltd, 2009:419-29.

29 Müller $\mathrm{E}, \mathrm{Schwameder} H$. Biomechanical aspects of new techniques in alpine skiing and ski-jumping. J Sports Sci 2003;21:679-92.

30 Petrone N, Johnson RJ, Shealy JE, et al. The effect of impact speed, construction, and layout of different ski safety barriers on peak decelerations and penetration values of a solid dummy during full scale impacts. Skiing Trauma Saf 2012;19:153-70.

31 Kröll J, Spörri J, Gilgien M, et al. Effect of ski geometry on aggressive ski behaviour and visual aesthetics: equipment designed to reduce risk of severe traumatic knee injuries in alpine giant slalom ski racing. $\mathrm{Br} J$ Sports $\mathrm{Med}$ 2016;50:21-6.

32 Spörri J, Kröll J, Gilgien $M$, et al. Sidecut radius and the mechanics of turningEquipment designed to reduce risk of severe traumatic knee injuries in alpine giant slalom ski racing. Br J Sports Med 2016;50:15-20.

33 Niessen W, Müller E. Carving-biomechanische Aspekte bei der Verwendung stark taillierter Skier und erhöhter Standflächen im alpinen Skisport. Leistungssport 1999;1:39-44. 